\title{
Pitman-Closeness of Preliminary Test and Some Classical Estimators Based on Records from Two-Parameter Exponential Distribution
}

\author{
Elham Mirfarah* and Jafar Ahmadi
}

Ferdowsi University of Mashhad

\begin{abstract}
In this paper, we study the performance of estimators of parameters of two-parameter exponential distribution based on upper records. The generalized likelihood ratio (GLR) test was used to generate preliminary test estimator (PTE) for both parameters. We have compared the proposed estimator with maximum likelihood (ML) and unbiased estimators (UE) under mean-squared error (MSE) and Pitman measure of closeness (PMC). Analytical as well as graphical methods are used to show the range of parameter in which PTE performs better than ML and UE. Results demonstrate that in the case of that prior information is not too far from its real value, the PTE is superior in compare with ML and UE based on both MSE and PMC criteria. The results of the paper will be useful in estimation with record data in life testing experiments.
\end{abstract}

Keywords. Preliminary test estimator; maximum likelihood estimator; unbiased estimator; mean-squared error; Pitman measure of closeness; record data.

MSC 2010: 62F10; 62F03.

\footnotetext{
* Corresponding author
} 


\section{Introduction}

The method known in the statistical literature as preliminary test estimation was implemented by Bancroft (1944). He dealt with the problem of estimating the unknown parameter when it is suspected that some "uncertain prior information" on the parameter of interest is available. This concept was developed by Han and Bancroft (1968) and since then several authors have considered estimation of the parameters based on PTE. For example, Chiou(1987) found the PTE of reliability in a life-testing model and Chiou (1988) utilized preliminary test estimation for the scale parameter of the extreme value distribution. Among the numerous papers discussing different aspects of PTE, one may refer to Benda (1996), Khan and Saleh (2001) and Hoque et al. (2009). Preliminary test methodology connects directly with the size of a test, so Kibria and Saleh (2006) suggested the optimum critical value for the test which formed PTE. Baklizi (2005) and Kibria and Saleh (2010) have applied the method of PTE for estimating the scale parameter of exponential distribution based on censored data. Arashi and Tabatabaey (2008) obtained preliminary test, Stein-type shrinkage and positive-rule shrinkage estimators, by assuming in the multiple regression model, that the error vector follows multivariate Student's t-distribution. Arashi (2012) proposed some estimators including PTE for the regression parameters of a multiple regression model and studied their performance. We refer the interested reader to Saleh (2006) for more details on the theory and application of PTE. Almost all of the works on PTE, utilized biased and risk function of estimators as a criteria of comparison between proposed estimators. In this paper, we consider the comparison of estimators based on PMC as well as MSE.

We recall that Pitman (1937) considered a comparison between two estimators of a parameter of interest based on the joint distribution of resulting losses. This was in contrast to usual decision-theory analysis based on the marginal distributions of the losses. According to his definition, if $T_{1}$ and $T_{2}$ are two estimators of a common parameter $\theta$, then $T_{1}$ is a Pitman closer estimator than $T_{2}$ if

$$
\operatorname{PMC}\left(T_{1}, T_{2} \mid \theta\right)=\operatorname{Pr}\left(\left|T_{1}-\theta\right|<\left|T_{2}-\theta\right|\right) \geqslant \frac{1}{2}, \quad \forall \theta \in \Omega,
$$

with strict inequality holding for at least one $\theta$, where $\Omega$ is the parameter space. Pitman made his original definition under the assumption that the probability of equality of competing estimators is zero. But what should 
we do with possible ties? i.e. $\operatorname{Pr}\left(T_{1}=T_{2}\right) \neq 0$. To overcome this problem, Kubokawa (1989) used the method, which later called the generalized Pitman closeness (GPC), as follows

$$
\operatorname{GPC}\left(T_{1}, T_{2} \mid \theta\right)=\operatorname{Pr}\left(\left|T_{1}-\theta\right|<\left|T_{2}-\theta\right|\right)-\operatorname{Pr}\left(\left|T_{1}-\theta\right|>\left|T_{2}-\theta\right|\right) .
$$

If $T_{1}$ and $T_{2}$ are two estimators of a common parameter $\theta$, then $T_{1}$ is corrected Pitman preferred to $T_{2}$, if

$$
\operatorname{GPC}\left(T_{1}, T_{2} \mid \theta\right) \geqslant 0, \quad \forall \theta \in \Omega,
$$

with strict inequality holding for at least one $\theta$. In the case of $\operatorname{Pr}\left(T_{1}=T_{2}\right)=$ 0 , the expressions (1) and (2) are equivalent. This concept has been studied in great detail in the literature, one may refer to the monograph by Keating et al. (1993) for comprehensive details about PMC. Among the few papers concerning PMC for comparing preliminarily test type estimators, Saleh and Sen (1991) used PMC and GPC to compare the preliminary test estimator (PTE), shrinkage and positive-rule versions of the MLE for location parameter of normal distribution. Let $X$ be a continuous random variable from exponential distribution with pdf

$$
f(x ; \mu, \sigma)=\sigma e^{-\sigma(x-\mu)}, \quad x \geqslant \mu, \sigma>0,
$$

where $\mu$ and $\sigma$ are location and scale parameters, respectively. The twoparameter exponential distribution has wide application in reliability theory, especially when there exists a lower threshold value of the variable. We also recall that, in a sequence of random variables $\left\{X_{n}, n \geqslant 1\right\}$, an observation $X_{j}$ is called a (upper) record if $X_{j}>X_{i}$ for all $i=1,2, \ldots, j-1$. We refer the reader to Arnold et al. (1998) for more details on the theory and applications of record data. Recently, PMC criterion faced considerable attention for comparing estimators based on different type of ordered data. Especially, Ahmadi and Balakrishnan (2009) and Raqab and Ahmadi (2011) discussed the Pitman-closeness of record values as estimator of population quantiles. Several estimation techniques exist in the literature for estimating the model parameters in terms of record values. However, the parameters estimation for the record data by using the preliminary test estimator is very few. In what follows, we consider the upper records from two-parameter exponential distribution and compute the MLE, UE and PTE of the scale and location parameters in order to see which one is Pitman closer to the target parameter. The layout of this paper is as follows. In Section 2, the problem of estimation 
has been discussed. We present the MLE, UE and PTE based on GLR test. Section 3 contains the PMC of estimators in pairs. Comparison based on MSE discussed with details in Section 4. Finally, numerical example is presented is Section 5 to show the performance of the estimators for real data.

\section{Preliminary Test Estimation}

In this section, we study the estimation of the location and scale parameters for the exponential distribution based on records, which include ML and UE, as well as PTE. Let us use the notation $R_{i}$ to signify the $i$ th upper record, for convenience of notations we consider $R_{1}=X_{1}$. Let $R_{1}, R_{2}, \ldots, R_{n}$ be the first $n$ upper records from exponential distribution with location parameter $\mu$ and scale parameter $\sigma$. Then, the likelihood function is given by (see, Arnold et al., 1998)

$$
L(\mu, \sigma)=\prod_{i=1}^{n-1} \frac{f\left(r_{i}\right)}{1-F\left(r_{i}\right)} f\left(r_{n}\right)=\sigma^{n} e^{-\sigma\left(r_{n}-\mu\right)}, \quad r_{1} \geqslant \mu,
$$

where $r_{i}$ is the corresponding observed value of $R_{i}$. Since the likelihood function is an increasing function of $\mu$, it is obvious that MLE of location parameter is $\hat{\mu}=R_{1}$. Also, with some straightforward calculation one can get that MLE of $\sigma$ is

$$
\hat{\sigma}=\frac{n}{R_{n}-R_{1}} .
$$

By using the properties of records from exponential distribution, it is known that $\sigma\left(R_{1}-\mu\right)$ and $\sigma\left(R_{n}-R_{1}\right)$ are independent with standard exponential and gamma distribution with parameters $(n-1,1)$. Therefore, UE of $\sigma$ and $\mu$ are

$$
\tilde{\sigma}=\frac{n-2}{R_{n}-R_{1}} \quad \text { and } \quad \tilde{\mu}=\frac{n R_{1}-R_{n}}{n-1},
$$

respectively.

Now our interest is to estimate the parameter when it is suspected that $\sigma$ (or $\mu$ ) may be $\sigma_{0}$ (or $\left.\mu_{0}\right)$. First, we consider the estimation of scale parameter. When the non sample prior information $\sigma=\sigma_{0}$ is doubted, the choice of estimator for $\sigma$ will depend on the outcome of the test $H_{0}: \sigma=\sigma_{0}$ against the alternative $H_{1}: \sigma \neq \sigma_{0}$. Combining the result of test and a target estimator, if $H_{0}$ were true we would use $\sigma_{0}$ as an estimator of $\sigma$, otherwise, 
PTE will reduce to the target estimator. In this context, UE is considered as target estimator. Accordingly, we write the possible estimator as

$$
\tilde{\sigma}^{P T}=\sigma_{0} I\left(A_{s}\right)+\tilde{\sigma} I\left(\bar{A}_{s}\right)=\tilde{\sigma}-\left(\tilde{\sigma}-\sigma_{0}\right) I\left(A_{s}\right),
$$

where $A_{s}$ is the acceptance region of $H_{0}: \sigma=\sigma_{0}$, vs. $H_{1}: \sigma \neq \sigma_{0}$ and $I\left(A_{s}\right)$ is the indicator function of set $A_{s}$. In order to determine the set $A_{s}$, we use the GLR test of level $\alpha$ where the likelihood ratio statistic is obtained as follows

$$
\Lambda=\frac{L\left(\hat{\mu}, \sigma_{0}\right)}{L(\hat{\mu}, \hat{\sigma})}=\left\{\frac{\sigma_{0}\left(R_{n}-R_{1}\right)}{n}\right\}^{n} e^{-n\left\{\frac{\sigma_{0}\left(R_{n}-R_{1}\right)}{n}-1\right\}}=\left(\frac{Z_{0}}{2 n}\right)^{n} e^{-n\left(\frac{Z_{0}}{2 n}-1\right)} .
$$

So the critical region of the GLR test is given by

$$
C_{s}=\left\{\mathbf{z}:\left(\frac{Z_{0}}{2 n}\right)^{n} e^{-n\left(\frac{Z_{0}}{2 n}-1\right)} \leqslant c_{0}\right\}
$$

where $c_{0}$ is chosen such that $\operatorname{Pr}\left\{\left(\frac{Z_{0}}{2 n}\right)^{n} e^{-n\left(\frac{Z_{0}}{2 n}-1\right)} \leqslant c_{0}\right\}=\alpha$. As a function of $Z_{0}$, the likelihood ratio statistic first increases and then decreases, so it can be clearly seen that we will reject $H_{0}$ if, for some positive values $a$ and $b$

$$
\left\{\begin{array}{l}
Z_{0}<a \quad \text { or } \quad Z_{0}>b, \\
a^{n} e^{-\frac{a}{2}}=b^{n} e^{-\frac{b}{2}}
\end{array}\right.
$$

Since under null hypothesis $Z_{0}$ has chi-square distribution with $\nu=2(n-1)$ degrees of freedom, we choose $a$ and $b$ such that

$$
\left\{\begin{array}{l}
\int_{a}^{b} h_{2(n-1)}(t) d t=1-\alpha \\
a^{2} h_{2(n-1)}(a)=b^{2} h_{2(n-1)}(b),
\end{array}\right.
$$

where $h_{\nu}(\cdot)$ stands for the pdf of chi-square distribution with $\nu=2(n-1)$ degrees of freedom. Table 680 of Tate and Klett (1959) presents critical values for applying the GLR test for some choices of $n$ and $\alpha$.

Similarly, to estimate the location parameter, we write the PTE as

$$
\tilde{\mu}^{P T}=\mu_{0} I\left(A_{l}\right)+\tilde{\mu} I\left(\bar{A}_{l}\right)=\tilde{\mu}-\left(\tilde{\mu}-\mu_{0}\right) I\left(A_{l}\right),
$$


where $A_{l}$ is the acceptance region of $H_{0}: \mu=\mu_{0}$, vs. $H_{1}: \mu \neq \mu_{0}$ and $I\left(A_{l}\right)$ is the indicator function of set $A_{l}$. To determine the set $A_{l}$, the GLR test of level $\alpha$ leads to the following quantity

$$
W=\frac{R_{1}-\mu_{0}}{\frac{R_{n}-R_{1}}{n-1}} .
$$

Using the independence of $\sigma\left(R_{1}-\mu_{0}\right)$ and $\sigma\left(R_{n}-R_{1}\right)$, it is easy to verify that $W$ has F-distribution with $(2,2(n-1))$ degrees of freedom (see, Arnold et al., 1998). We will reject $H_{0}: \mu=\mu_{0}$ if

$$
\frac{(n-1)\left(R_{1}-\mu_{0}\right)}{R_{n}-R_{1}} \geqslant f_{(2,2(n-1))}(1-\alpha)=f
$$

where $f_{\left(\nu_{1}, \nu_{2}\right)}(p)$ is the $p$ th quantile of the F-distribution with $\left(\nu_{1}, \nu_{2}\right)$ degrees of freedom.

In the sequence, the proposed estimators will be compared under PMC and MSE criteria.

\section{Comparison Based on PMC}

In this section, we compare the proposed estimators via Pitman measure of closeness. First, the estimators of scale parameter are compared in pairs, and then we consider the estimators of the location parameter.

\subsection{Results for Scale Parameter}

In this subsection, the comparison of scale estimators is considered in terms of Pitman measure of closeness. Since PMC suffers from lack of transitivity, we have to conduct three comparisons. To this end, we use the variable $Z=2 \sigma\left(R_{n}-R_{1}\right)$ and define the following events

$$
\begin{gathered}
A_{s}=\left\{\frac{a}{\Delta_{s}}<Z<\frac{b}{\Delta_{s}}\right\}, \\
B_{1}=\{Z<2(n-1)\} \text { and } B_{2(j)}=\left\{\left|\Delta_{s}-1\right|<\left|\frac{2 j}{Z}-1\right|\right\} .
\end{gathered}
$$

where $\Delta_{s}=\frac{\sigma_{0}}{\sigma}$ and $j=n-2, n$. We will show that the Pitman-closeness probability of the estimators is the intersection or union of this events. Let 
us first find the probability of desired events. It is easy to verify that

$\operatorname{Pr}\left(B_{1} \cap \bar{A}_{s}\right)=\operatorname{Pr}\left(Z<\min \left\{2(n-1), \frac{a}{\Delta_{s}}\right\}\right)+\operatorname{Pr}\left(Z<2(n-1), Z>\frac{b}{\Delta_{s}}\right)$

With some simple calculations, it follows that the event $B_{2(j)}$ is equivalent to

$$
B_{2(j)}= \begin{cases}\left\{Z<\frac{2 j}{2-\Delta_{s}}\right\} \cup\left\{Z>\frac{2 j}{\Delta_{s}}\right\}, & 0<\Delta_{s}<1 ; \\ \left\{Z<\frac{2 j}{\Delta_{s}}\right\} \cup\left\{Z>\frac{2 j}{2-\Delta_{s}}\right\}, & 1 \leqslant \Delta_{s}<2 ; \\ \left\{Z<\frac{2 j}{\Delta_{s}}\right\}, & 2 \leqslant \Delta_{s} .\end{cases}
$$

So the probability of the intersection of $B_{2(j)}$ and $A_{s}$ is obtained as

$$
\begin{aligned}
& \operatorname{Pr}\left(B_{2(j)} \cap A_{s}\right)= \\
& \begin{cases}\operatorname{Pr}\left\{\frac{a}{\Delta_{s}}<Z<\min \left(\frac{b}{\Delta_{s}}, \frac{2 j}{2-\Delta_{s}}\right)\right\}+\operatorname{Pr}\left\{\frac{2 j}{\Delta_{s}}<Z<\frac{b}{\Delta_{s}}\right\}, & 0<\Delta_{s}<1 ; \\
\operatorname{Pr}\left\{\frac{a}{\Delta_{s}}<Z<\frac{2 j}{\Delta_{s}}\right\}+\operatorname{Pr}\left\{\max \left(\frac{a}{\Delta_{s}}, \frac{2 j}{2-\Delta_{s}}\right)<Z<\frac{b}{\Delta_{s}}\right\}, & 1 \leqslant \Delta_{s}<2 ; \\
\operatorname{Pr}\left\{\frac{a}{\Delta_{s}}<Z<\frac{2 j}{\Delta_{s}}\right\}, & 2 \leqslant \Delta_{s} .\end{cases}
\end{aligned}
$$

If $0<\Delta_{s}<1$, then $\frac{b}{\Delta_{s}}>\frac{2 j}{2-\Delta_{s}}$, however in the case of $\frac{a}{\Delta_{s}}>\frac{2 j}{2-\Delta_{s}}$, we have

$$
\operatorname{Pr}\left\{\frac{a}{\Delta_{s}}<Z<\min \left(\frac{b}{\Delta_{s}}, \frac{2 j}{2-\Delta_{s}}\right)\right\}=0
$$

And similarly, if $1<\Delta_{s}<2$, then $\frac{a}{\Delta_{s}}<\frac{2 j}{2-\Delta_{s}}$, but $\frac{b}{\Delta_{s}}<\frac{2 j}{2-\Delta_{s}}$ leads to

$$
\operatorname{Pr}\left\{\max \left(\frac{a}{\Delta_{s}}, \frac{2 j}{2-\Delta_{s}}\right)<Z<\frac{b}{\Delta_{s}}\right\}=0
$$


Therefore, the probability of $\left(B_{2(j)} \cap A_{s}\right)$ can be rewritten as follows

$$
\begin{aligned}
& \operatorname{Pr}\left(B_{2(j)} \cap A_{s}\right)= \\
& \begin{cases}\operatorname{Pr}\left\{\min \left(\frac{a}{\Delta_{s}}, \frac{2 j}{2-\Delta_{s}}\right)<Z<\frac{2 j}{2-\Delta_{s}}\right\}+\operatorname{Pr}\left\{\frac{2 j}{\Delta_{s}}<Z<\frac{b}{\Delta_{s}}\right\}, & 0<\Delta_{s}<1 \\
\operatorname{Pr}\left\{\frac{a}{\Delta_{s}}<Z<\frac{2 j}{\Delta_{s}}\right\}+\operatorname{Pr}\left\{\frac{2 j}{2-\Delta_{s}}<Z<\max \left(\frac{2 j}{2-\Delta_{s}}, \frac{b}{\Delta_{s}}\right)\right\}, & 1 \leqslant \Delta_{s}<2 \\
\operatorname{Pr}\left\{\frac{a}{\Delta_{s}}<Z<\frac{2 j}{\Delta_{s}}\right\}, & 2 \leqslant \Delta_{s} .\end{cases}
\end{aligned}
$$

With the same method as mentioned for $\operatorname{Pr}\left(B_{2(j)} \cap A_{s}\right)$, we have

$$
\operatorname{Pr}\left(\bar{B}_{2(j)} \cap A_{s}\right)= \begin{cases}\operatorname{Pr}\left\{\max \left(\frac{a}{\Delta_{s}}, \frac{2 j}{2-\Delta_{s}}\right)<Z<\frac{2 j}{\Delta_{s}}\right\}, & 0<\Delta_{s}<1 ; \\ \operatorname{Pr}\left\{\frac{2 j}{\Delta_{s}}<Z<\min \left(\frac{b}{\Delta_{s}}, \frac{2 j}{2-\Delta_{s}}\right)\right\}, & 1 \leqslant \Delta_{s}<2 ; \\ \operatorname{Pr}\left\{\frac{2 j}{\Delta_{s}}<Z<\frac{b}{\Delta_{s}}\right\}, & 2 \leqslant \Delta_{s} .\end{cases}
$$

Now, we turn our attention to the probability of closeness of the scale estimators. For the calculation of the probability of closeness between $\hat{\sigma}$ and $\tilde{\sigma}$, we use (1) as follows

$$
\begin{aligned}
\operatorname{PMC}(\tilde{\sigma}, \hat{\sigma} \mid \sigma) & =\operatorname{Pr}\left\{\left|\frac{(n-2)}{\left(R_{n}-R_{1}\right)}-\sigma\right|<\left|\frac{n}{\left(R_{n}-R_{1}\right)}-\sigma\right|\right\} \\
& =\operatorname{Pr}\left\{(n-2)^{2}-2 \sigma(n-2)\left(R_{n}-R_{1}\right)<n^{2}-2 n \sigma\left(R_{n}-R_{1}\right)\right\} \\
& =\operatorname{Pr}\{Z<2(n-1)\} .
\end{aligned}
$$

With the same justification as mentioned in Balakrishnan et al. (2011), we have

$$
\operatorname{PMC}(\tilde{\sigma}, \hat{\sigma} \mid \sigma)=\operatorname{Pr}\left(B_{1}\right) \geqslant \frac{1}{2} .
$$

So the unbiased estimator of $\sigma$ is always Pitman closer than the corresponding MLE. Now, we consider the comparison of $\tilde{\sigma}^{P T}$ with UE. Since

$$
\operatorname{Pr}\left(\tilde{\sigma}^{P T}=\tilde{\sigma}\right)=\operatorname{Pr}\left(Z<\frac{a}{\Delta_{s}} \text { or } Z>\frac{b}{\Delta_{s}}\right)=\operatorname{Pr}\left(\bar{A}_{s}\right) \neq 0,
$$


comparison is applied based on (2). Thus, we have

$$
\begin{aligned}
\operatorname{GPC}\left(\tilde{\sigma}^{P T}, \tilde{\sigma} \mid \sigma\right) & =\operatorname{Pr}\left(\left|\tilde{\sigma}^{P T}-\sigma\right|<|\tilde{\sigma}-\sigma|\right)-\operatorname{Pr}\left(\left|\tilde{\sigma}^{P T}-\sigma\right|>|\tilde{\sigma}-\sigma|\right) \\
& =\operatorname{Pr}\left(B_{2(n-2)} \cap A_{s}\right)-\operatorname{Pr}\left(\bar{B}_{2(n-2)} \cap A_{s}\right) .
\end{aligned}
$$

Substituting (4) and (5), for $j=n-2$, in (6), we are able to compare two estimators. Let us consider the Pitman closeness of $\tilde{\sigma}^{P T}$ in compare with MLE. In this case,

$$
\operatorname{Pr}\left\{\tilde{\sigma}-\left(\tilde{\sigma}-\sigma_{0}\right) I\left(A_{s}\right)=\hat{\sigma}\right\}=0,
$$

so we use the concept of PMC to compare the estimators. We have

$$
\begin{aligned}
\operatorname{PMC}\left(\tilde{\sigma}^{P T}, \hat{\sigma} \mid \sigma\right)= & \operatorname{Pr}\left\{\left|\tilde{\sigma}-\left(\tilde{\sigma}-\sigma_{0}\right) I\left(A_{s}\right)-\sigma\right|<|\hat{\sigma}-\sigma|\right\} \\
= & \operatorname{Pr}\left\{|\tilde{\sigma}-\sigma|<|\hat{\sigma}-\sigma|, I\left(A_{s}\right)=0\right\} \\
& +\operatorname{Pr}\left\{\left|\sigma_{0}-\sigma\right|<|\hat{\sigma}-\sigma|, I\left(A_{s}\right)=1\right\} \\
= & \operatorname{Pr}\left\{B_{1} \cap \bar{A}_{s}\right\}+\operatorname{Pr}\left\{B_{2(n)} \cap A_{s}\right\},
\end{aligned}
$$

which could be found by (3) and (4). All the probabilities of closeness depend on the number of records, $\Delta_{s}$ and the size of test, so for different values of $n$ and $\alpha$, the graphs of GPC and PMC have been drawn for $\Delta_{s} \in(0,3)$, in Figures 1 and 2. To determine the range of $\Delta_{s}$ for which $\tilde{\sigma}^{P T}$ is closer to $\sigma$, the line $\mathrm{GPC}=0$ (or PMC $=0.5$ ) is drawn. In plot $(\mathrm{A})$ of Figures 1 and 2 , the GPC or PMC is drawn for fixed $n=10$ and $\alpha=0.05,0.025,0.005,0.0025$, and plot (B), the GPC or PMC is drawn for fixed $\alpha=0.05$ and $n=4,5,6,7$. The maximum value of PMC or GPC occurred near the null hypothesis.

\subsection{Results for Location Parameter}

In this subsection, we compare three estimators of location parameter, which was presented in Section 2. We follow the same layout as Subsection 3.1, i.e. we first defined some events and find their probabilities, then the probabilities of Pitman closeness are obtained using these notations. Let $U=\sigma\left(R_{1}-\mu\right)$ and $V=\sigma\left(R_{n}-R_{1}\right)$, we consider

$$
\begin{gathered}
A_{l}=\left\{(n-1)\left(U+\Delta_{l}\right)<f V\right\}, \quad D_{1}=\left\{2 U>\frac{V}{n-1}\right\}, \\
D_{2}=\left\{\left|\Delta_{l}\right|<U\right\} \quad \text { and } \quad D_{3}=\left\{\left|\Delta_{l}\right|<\left|U-\frac{V}{n-1}\right|\right\},
\end{gathered}
$$



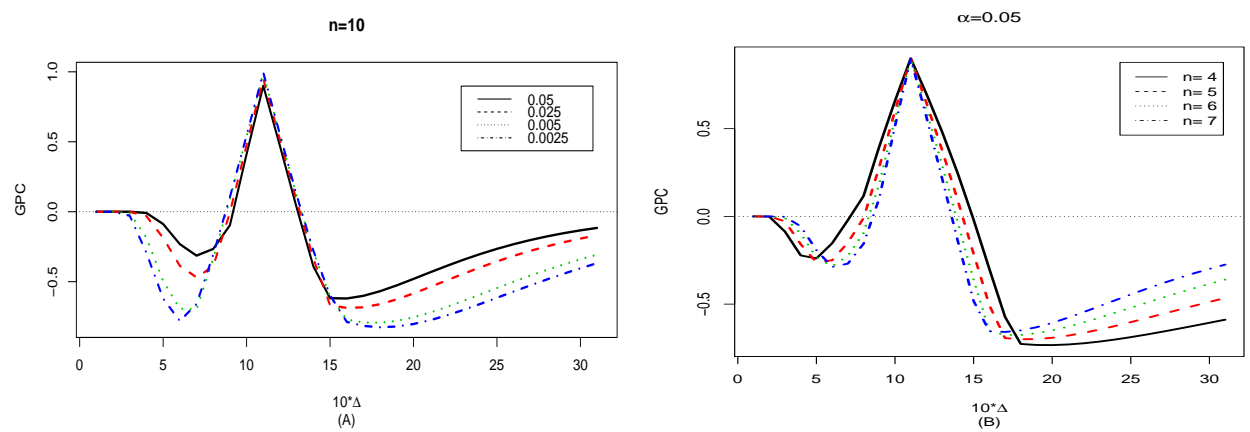

Figure 1. GPC of $\tilde{\sigma}^{P T}$ relative to $\tilde{\sigma}$ : (A) for fixed $n=10,(\mathrm{~B})$ for fixed $\alpha=0.05$.
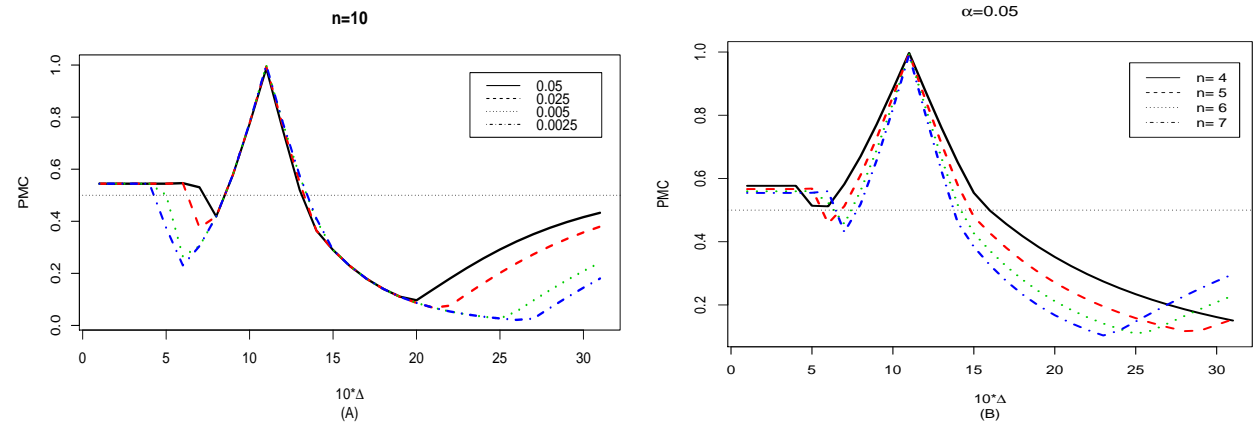

Figure 2. PMC of $\tilde{\sigma}^{P T}$ relative to $\hat{\sigma}$ : (A) for fixed $n=10$, (B) for fixed $\alpha=0.05$.

where $\Delta_{l}=\sigma\left(\mu-\mu_{0}\right)$ and $f=f_{(2,2(n-1))}(1-\alpha)$. For comparison of estimators, we need the probabilities of the mentioned events. For later use, let us take $t_{1}=\frac{\Delta_{l}(n-1)}{f}, t_{2}=\frac{2 \Delta_{l}(n-1)}{f-1}, t_{3}=\frac{\Delta_{l}(n-1)}{f-0.5}, \lambda_{1}=1+\frac{f}{n-1}$ and

$$
\rho_{n}(t, \lambda)=\int_{t}^{\infty} \frac{y^{n-1} e^{-\lambda y}}{\Gamma(n)} d y=\frac{1}{\lambda^{n}} \sum_{k=0}^{n-1} \frac{e^{-\lambda t}(\lambda t)^{k}}{k !}
$$

We start with the probability of intersection of $D_{1}$ and $\bar{A}_{l}$. In this case, we have two situations. For $\Delta_{l} \leqslant 0$, since $\frac{V}{2(n-1)}<\frac{f V}{n-1}$ with probability one, 
we find that

$$
\begin{aligned}
\operatorname{Pr}\left\{D_{1} \cap \bar{A}_{l}\right\} & =\operatorname{Pr}\left\{U>\frac{V}{2(n-1)}, U>\frac{f V}{n-1}-\Delta_{l}\right\} \\
& =\operatorname{Pr}\left(U>\frac{f V}{n-1}-\Delta_{l}\right) \\
& =\int_{0}^{\infty} \int_{\frac{f v}{n-1}-\Delta_{l}}^{\infty} \frac{v^{n-2}}{\Gamma(n-1)} e^{-(u+v)} d u d v=e^{\Delta_{l}} \lambda_{1}^{1-n} .
\end{aligned}
$$

And for $\Delta_{l}>0$, the probability of $D_{1} \cap \bar{A}_{l}$ is obtained as

$$
\begin{aligned}
\operatorname{Pr}\left\{D_{1} \cap \bar{A}_{l}\right\} & =\operatorname{Pr}\left(U>\max \left\{\frac{V}{2(n-1)}, \frac{f V}{n-1}-\Delta_{l}\right\}\right) \\
& =\int_{0}^{t_{3}} \frac{v^{n-2}}{\Gamma(n-1)} e^{-v \frac{2 n-1}{2(n-1)}} d v+e^{\Delta_{l}} \int_{t_{3}}^{\infty} \frac{v^{n-2}}{\Gamma(n-1)} e^{-v\left(\lambda_{1}\right)} d v \\
& =\left\{\frac{2(n-1)}{2 n-1}\right\}^{n-1}-\rho_{n-1}\left\{t_{3}, \frac{2 n-1}{2(n-1)}\right\}+e^{\Delta_{l}} \rho_{n-1}\left(t_{3}, \lambda_{1}\right),
\end{aligned}
$$

where $\rho_{n}(\cdot, \cdot)$ is defined in $(7)$. For the probability of the second event, i.e. $D_{2}$, we consider two cases, first, for $\Delta_{l} \leqslant 0$, we have

$$
\begin{aligned}
\operatorname{Pr}\left\{D_{2} \cap A_{l}\right\} & =\operatorname{Pr}\left(\left|\Delta_{l}\right|<U, U<\frac{f}{n-1} V-\Delta_{l}\right) \\
& =\int_{0}^{\infty} \int_{\left|\Delta_{l}\right|}^{\frac{f}{n-1} v-\Delta_{l}} \frac{v^{n-2}}{\Gamma(n-1)} e^{-(u+v)} d u d v=e^{\Delta_{l}}\left\{1-\lambda_{1}^{1-n}\right\} .
\end{aligned}
$$

And for $\Delta_{l}>0$, the probability of $D_{2} \cap A_{l}$ is given by

$$
\begin{aligned}
\operatorname{Pr}\left\{D_{2} \cap A_{l}\right\} & =\int_{2 t_{1}}^{\infty} \int_{\Delta_{l}}^{\frac{f}{n-1} v-\Delta_{l}} \frac{v^{n-2}}{\Gamma(n-1)} e^{-(u+v)} d u d v \\
& =e^{-\Delta_{l}} \rho_{n-1}\left(2 t_{1}, 1\right)-e^{\Delta_{l}} \rho_{n-1}\left(2 t_{1}, \lambda_{1}\right) .
\end{aligned}
$$

Now, let us find the probability of $D_{3} \cap A_{l}$. We have

$$
\operatorname{Pr}\left\{D_{3} \cap A_{l}\right\}=\operatorname{Pr}\left(\left|U-\frac{V}{n-1}\right|>\left|\Delta_{l}\right|, U<\frac{f}{n-1} V-\Delta_{l}\right) .
$$


For $\Delta_{l}>0$, the right hand side of (12) is equal to

$$
\begin{aligned}
e^{-\Delta_{l}} \rho_{n-1}\left(t_{2}, \frac{n}{n-1}\right)-e^{\Delta_{l}} \rho_{n-1}\left(t_{2}, \lambda_{1}\right)+\rho_{n-1}\left(\Delta_{l}(n-1), 1\right)-e^{\Delta_{l}} & \\
& \times \rho_{n-1}\left(\Delta_{l}(n-1), \frac{n}{n-1}\right),
\end{aligned}
$$

and, for $\Delta_{l} \leqslant 0$, the right hand side of (12) is equal to

$$
\begin{aligned}
e^{\Delta_{l}}\left\{\left(\frac{n-1}{n}\right)^{n-1}-\left(\frac{1}{\lambda_{1}}\right)^{n-1}\right\}+\rho_{n-1}\left(-\Delta_{l}(n\right. & -1), 1)-e^{-\Delta_{l}} \\
& \times \rho_{n-1}\left(-\Delta_{l}(n-1), \frac{n}{n-1}\right) .
\end{aligned}
$$

Finally, the probability of $\bar{D}_{3} \cap A_{l}$ is given by

$$
\begin{aligned}
\operatorname{Pr}\left\{\bar{D}_{3} \cap A_{l}\right\}= & \operatorname{Pr}\left(\left|\Delta_{l}\right|>\left|U-\frac{V}{n-1}\right|, U<\frac{f}{n-1} V-\Delta_{l}\right) \\
= & 1-\rho_{n-1}\left(-\Delta_{l}(n-1), 1\right)-\frac{e^{\Delta_{l}}}{\left(\frac{n}{n-1}\right)^{n-1}} \\
& +e^{-\Delta_{l}} \rho_{n-1}\left(-\Delta_{l}(n-1), \frac{n}{n-1}\right),
\end{aligned}
$$

when $\Delta_{l} \leqslant 0$, and it is equal to

$$
\begin{aligned}
\operatorname{Pr}\left\{\bar{D}_{3} \cap A_{l}\right\}= & \left(e^{\Delta_{l}}-e^{-\Delta_{l}}\right)\left\{\rho_{n-1}\left(\Delta_{l}(n-1), \frac{n}{n-1}\right)\right\}-\rho_{n-1}\left(\Delta_{l}(n-1), 1\right) \\
& -e^{-\Delta_{l}}\left\{\rho_{n-1}\left(t_{2}, \frac{n}{n-1}\right)-\rho_{n-1}\left(\Delta_{l}(n-1), \frac{n}{n-1}\right)\right\} \\
& \left.+\rho_{n-1}\left(t_{1}, 1\right)-e^{\Delta_{l}}\left\{\rho_{n-1}\left(t_{1}, \lambda_{1}\right)-\rho_{n-1}\left(t_{2}, \lambda_{1}\right)\right]\right\}
\end{aligned}
$$

when $\Delta_{l}>0$. Now, we turn our attention to the probability of closeness of location estimators. To compare the estimator $\tilde{\mu}$ with $\hat{\mu}$, in terms of PMC, we have

$$
\operatorname{PMC}(\tilde{\mu}, \hat{\mu} \mid \mu)=\operatorname{Pr}\left(D_{1}\right)=\operatorname{Pr}\left(2 U>\frac{V}{n-1}\right) .
$$


On the other hand, it is easy to find that

$$
\begin{aligned}
\operatorname{Pr}\left\{D_{1}\right\} & =\operatorname{Pr}\left(2 U>\frac{V}{n-1}\right) \\
& =\int_{0}^{\infty} \int_{\frac{v}{2(n-1)}}^{\infty} \frac{v^{n-2}}{\Gamma(n-1)} e^{-(u+v)} d u d v \\
& =\left\{\frac{2(n-1)}{2 n-1}\right\}^{n-1} .
\end{aligned}
$$

Since $\operatorname{Pr}\left\{D_{1}\right\}$ is decreasing in $n$, and

$$
\lim _{n \rightarrow \infty}\left\{\frac{2(n-1)}{2 n-1}\right\}^{n-1}>\frac{1}{2},
$$

the UE of $\mu$ is always Pitman closer than MLE. Now, we consider the comparison of $\tilde{\mu}^{P T}$ with UE of $\mu$. Since

$$
\operatorname{Pr}\left\{\tilde{\mu}^{P T}=\tilde{\mu}\right\}=\operatorname{Pr}\left((n-1)\left(U+\Delta_{l}\right)>f V\right)=\operatorname{Pr}\left(\bar{A}_{l}\right) \neq 0,
$$

comparison is applied based on GPC. Thus,

$$
\begin{aligned}
\operatorname{GPC}\left(\tilde{\mu}^{P T}, \tilde{\mu} \mid \mu\right) & =\operatorname{Pr}\left(\left|\tilde{\mu}^{P T}-\mu\right|<|\tilde{\mu}-\mu|\right)-\operatorname{Pr}\left(\left|\tilde{\mu}^{P T}-\mu\right|>|\tilde{\mu}-\mu|\right) \\
& =\operatorname{Pr}\left(D_{3} \cap A_{l}\right)-\operatorname{Pr}\left(\bar{D}_{3} \cap A_{l}\right) .
\end{aligned}
$$

Using (12), (13) and (14), we are able to compare the estimators. Let us consider the Pitman closeness of $\tilde{\mu}^{P T}$ in compare with MLE. In this case

$$
\operatorname{Pr}\left\{\tilde{\mu}-\left(\tilde{\mu}-\mu_{0}\right) I\left(A_{l}\right)=\hat{\mu}\right\}=0,
$$

so, we use the concept of PMC to compare the estimators.

$$
\begin{aligned}
\operatorname{PMC}\left(\tilde{\mu}^{P T}, \hat{\mu} \mid \mu\right)= & \operatorname{Pr}\left\{\left|\tilde{\mu}-\left(\tilde{\mu}-\mu_{0}\right) I\left(A_{l}\right)-\mu\right|<|\hat{\mu}-\mu|\right\} \\
= & \operatorname{Pr}\left\{2 R_{1}-\frac{R_{n}-R_{1}}{n-1}>2 \mu, I\left(A_{l}\right)=0\right\} \\
& +\operatorname{Pr}\left\{\left|\mu_{0}-\mu\right|<\left|R_{1}-\mu\right|, I\left(A_{l}\right)=1\right\} \\
= & \operatorname{Pr}\left\{D_{1} \cap \bar{A}_{l}\right\}+\operatorname{Pr}\left\{D_{2} \cap A_{l}\right\} .
\end{aligned}
$$


Using (8), (9), (10) and (11), we find the $\operatorname{PMC}\left(\tilde{\mu}^{P T}, \hat{\mu} \mid \mu\right)$.

Similar to the probability of closeness for the scale estimators, in this section all the probabilities depend on the number of records, $\Delta_{l}$ and the size of test. The graphs of GPC and PMC for different values of $n$ and $\alpha$, when $\Delta_{l} \in(-5,5)$ are displayed in Figures 3 and 4, respectively. Again the maximum value of GPC and PMC occurred near the null hypothesis. Looking at the Figures 3 and 4, it is noticeable that Pitman criterion seems robust for location parameter.
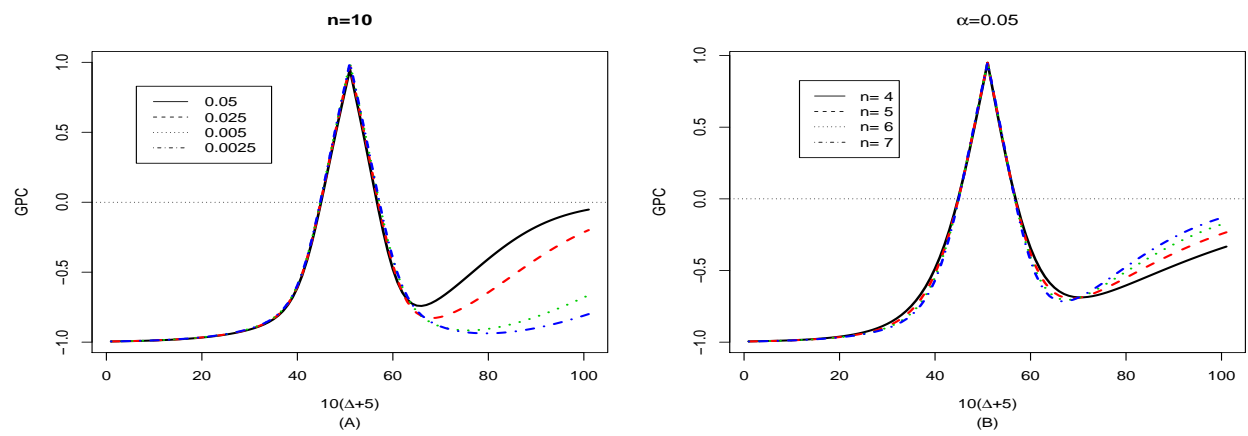

Figure 3. GPC of $\tilde{\mu}^{P T}$ relative to $\tilde{\mu}$ : (A) for fixed $n=10,(\mathrm{~B})$ for fixed $\alpha=0.05$.
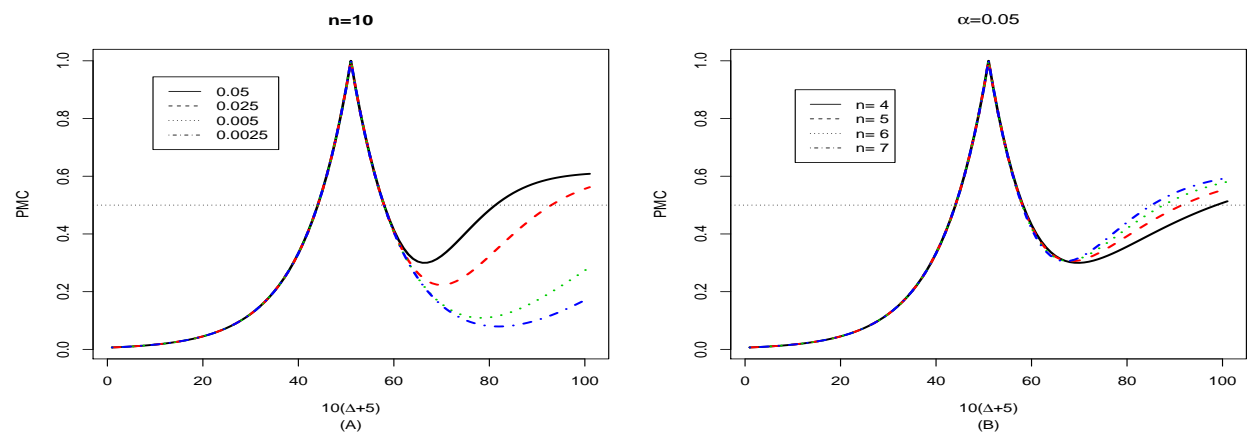

Figure 4. PMC of $\tilde{\mu}^{P T}$ relative to $\hat{\mu}$ : (A) for fixed $n=10$, (B) for fixed $\alpha=0.05$. 


\section{MSE Evaluations}

Considering the MSE as a criterion of comparison, in this section we compare the performance of the proposed estimators based on mean-squared relative efficiency (MRE). We first consider the estimators of the scale parameter and then will find the MRE of the location estimators.

\subsection{Results for Scale Parameter}

In order to derive the MSE of estimators, we recall that if $\chi_{\nu}^{2}$ is a chi-square random variable with $\nu$ degrees of freedom, then, for $k>0$

$$
E\left\{\left(\chi_{\nu}^{2}\right)^{-k}\right\}=\left(\frac{1}{2}\right)^{k} \frac{\Gamma\left(\frac{\nu}{2}-k\right)}{\Gamma\left(\frac{\nu}{2}\right)},
$$

where $\Gamma(\cdot)$ is the complete gamma function. Moreover, let $\varphi\left(\chi_{\nu}^{2}\right)$ be a measurable function of $\chi_{\nu}^{2}$, then

$$
E\left\{\left(\chi_{\nu}^{2}\right)^{-k} \varphi\left(\chi_{\nu}^{2}\right)\right\}=\left(\frac{1}{2}\right)^{k} \frac{\Gamma\left(\frac{\nu}{2}-k\right)}{\Gamma\left(\frac{\nu}{2}\right)} E\left\{\varphi\left(\chi_{\nu-2 k}^{2}\right)\right\}
$$

From (15), MSE of ML and unbiased estimators are straightforward. We have

$$
M S E(\hat{\sigma})=\frac{(n+6) \sigma^{2}}{(n-2)(n-3)} \text { and } M S E(\tilde{\sigma})=\frac{\sigma^{2}}{(n-3)} .
$$

Now, for PTE we have

$$
\begin{aligned}
M S E\left(\tilde{\sigma}^{P T}\right)= & E\left\{(\tilde{\sigma}-\sigma)-\left(\tilde{\sigma}-\sigma_{0}\right) I\left(A_{s}\right)\right\}^{2} \\
= & M S E(\tilde{\sigma})-E\left\{\tilde{\sigma}^{2} I\left(A_{s}\right)\right\}+\left(\sigma_{0}^{2}-2 \sigma \sigma_{0}\right) E\left\{I\left(A_{s}\right)\right\} \\
& +2 \sigma E\left\{\tilde{\sigma} I\left(A_{s}\right)\right\} .
\end{aligned}
$$

The first term on the right hand side of (18) is given as in (17). For the second term, take $\varphi\left(\chi_{\nu}^{2}\right)=I\left(A_{s}\right)$ then using (16), for any $k>0$, we have

$$
E\left\{\tilde{\sigma}^{k} I\left(A_{s}\right)\right\}=\{\sigma(n-2)\}^{k} \frac{\Gamma\left(\frac{\nu}{2}-k\right)}{\Gamma\left(\frac{\nu}{2}\right)}\left\{H_{\nu-2 k}\left(\frac{b}{\Delta_{s}}\right)-H_{\nu-2 k}\left(\frac{a}{\Delta_{s}}\right)\right\},
$$


where $\Delta_{s}=\frac{\sigma_{0}}{\sigma}$ and $H_{\nu}(\cdot)$ stands for the cdf of chi-square distribution with $\nu$ degrees of freedom. For $k=0,1,2$, substitution (19) in (18) leads to

$$
\begin{aligned}
M S E\left(\tilde{\sigma}^{P T}\right)= & M S E(\tilde{\sigma})-\frac{\sigma^{2}(n-2)\left\{H_{\nu-4}\left(\frac{b}{\Delta_{s}}\right)-H_{\nu-4}\left(\frac{a}{\Delta_{s}}\right)\right\}}{(n-3)}+\left(\sigma_{0}^{2}-2 \sigma \sigma_{0}\right) \\
& \times\left\{H_{\nu}\left(\frac{b}{\Delta_{s}}\right)-H_{\nu}\left(\frac{a}{\Delta_{s}}\right)\right\} \\
& +2 \sigma^{2}\left\{H_{\nu-2}\left(\frac{b}{\Delta_{s}}\right)-H_{\nu-2}\left(\frac{a}{\Delta_{s}}\right)\right\} .
\end{aligned}
$$

The efficiency of MLE compare to UE is

$$
\operatorname{MRE}(\tilde{\sigma}, \hat{\sigma})=\frac{M S E(\hat{\sigma})}{M S E(\tilde{\sigma})}=\frac{n+6}{n-2}>1 .
$$

Therefore, based on MSE criteria unbiased estimator of the scale parameter is superior. Now, we compare the MSE functions of $\hat{\sigma}$ and $\tilde{\sigma}$ with respect to preliminary test estimator.

$$
\begin{aligned}
\operatorname{MRE}\left(\tilde{\sigma}^{P T}, \tilde{\sigma}\right)= & {\left[1-(n-2)\left\{H_{\nu-4}\left(\frac{b}{\Delta_{s}}\right)-H_{\nu-4}\left(\frac{a}{\Delta_{s}}\right)\right\}\right.} \\
& +(n-3)\left(\Delta_{s}^{2}-2 \Delta_{s}\right)\left\{H_{\nu}\left(\frac{b}{\Delta_{s}}\right)-H_{\nu}\left(\frac{a}{\Delta_{s}}\right)\right\} \\
& \left.+2(n-3)\left\{H_{\nu-2}\left(\frac{b}{\Delta_{s}}\right)-H_{\nu-2}\left(\frac{a}{\Delta_{s}}\right)\right\}\right]^{-1} .
\end{aligned}
$$

Let us now consider the efficiency function of the $\tilde{\sigma}^{P T}$ relative to the MLE. Therefore,

$$
\begin{aligned}
\operatorname{MRE}\left(\tilde{\sigma}^{P T}, \hat{\sigma}\right)= & {\left[\frac{(n-2)}{(n+6)}-\frac{(n-2)^{2}}{(n+6)}\left\{H_{\nu-4}\left(\frac{b}{\Delta_{s}}\right)-H_{\nu-4}\left(\frac{a}{\Delta_{s}}\right)\right\}\right.} \\
& +\frac{(n-2)(n-3)}{(n+6)}\left(\Delta_{s}^{2}-2 \Delta_{s}\right)\left\{H_{\nu}\left(\frac{b}{\Delta_{s}}\right)-H_{\nu}\left(\frac{a}{\Delta_{s}}\right)\right\} \\
& \left.+\frac{2(n-2)(n-3)}{(n+6)}\left\{H_{\nu-2}\left(\frac{b}{\Delta_{s}}\right)-H_{\nu-2}\left(\frac{a}{\Delta_{s}}\right)\right\}\right]^{-1} .
\end{aligned}
$$


Note that the relative efficiencies depend on the number of records and size of the test. We have plotted the MRE of the proposed estimators for fixed $n=10$ and $\alpha=0.05,0.025,0.005,0.0025$, in part (A) of Figures 5 and 6. In plot (B) of these figures, MREs are presented for fixed $\alpha=0.05$ and $n=4,5,6,7$. Obviously, the relative efficiency reaches its maximum under $H_{0}$. The preliminary test estimator is more efficient near the null hypothesis, which was widely anticipated. Looking at the plot (A) of these figures, it is noticeable that comparing with MSE, PMC criterion is less effected by the size of test.
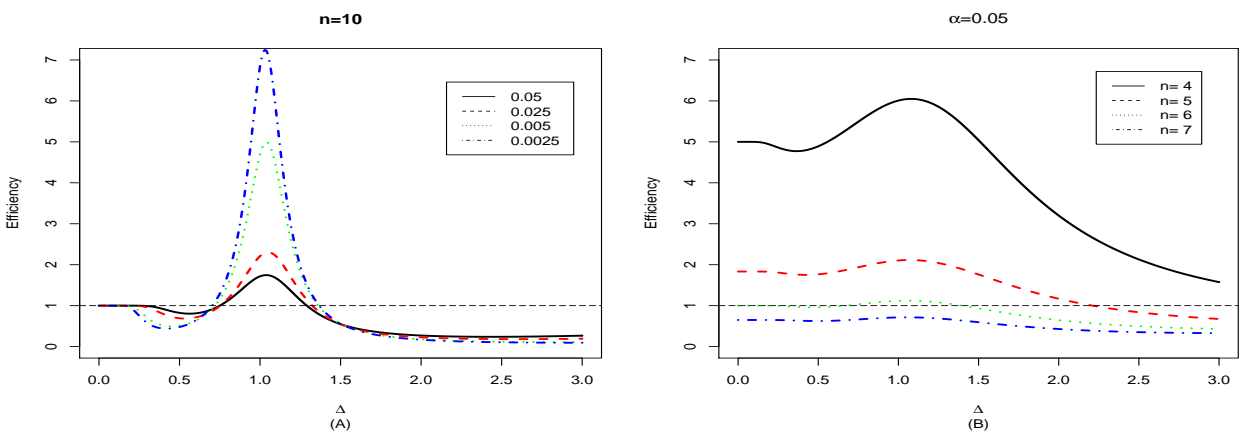

Figure 5. Efficiency of $\tilde{\sigma}^{P T}$ relative to $\tilde{\sigma}$ : (A) for fixed $n=10$, (B) for fixed $\alpha=0.05$.
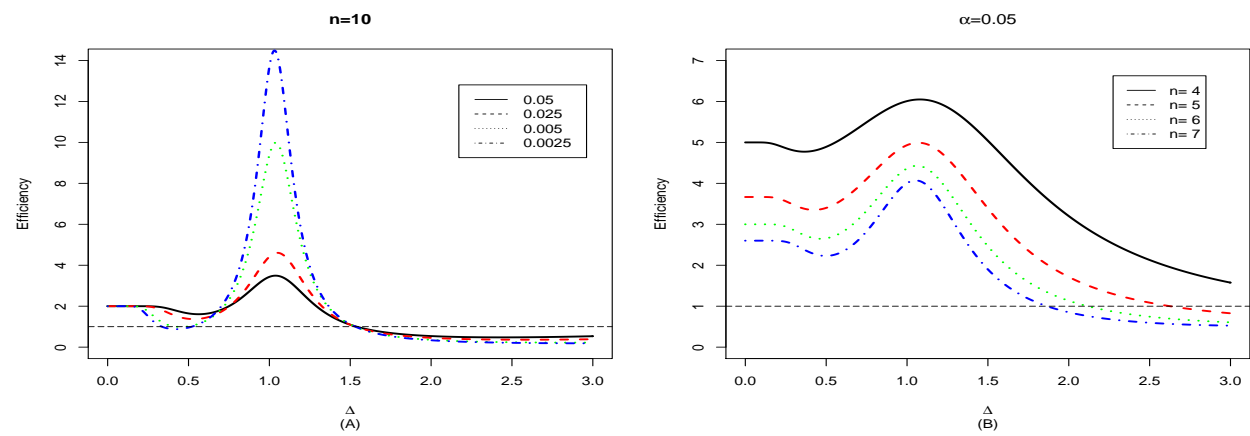

Figure 6. Efficiency of $\tilde{\sigma}^{P T}$ relative to $\hat{\sigma}$ : (A) for fixed $n=10$, (B) for fixed $\alpha=0.05$. 


\subsection{Results for Location Parameter}

In this subsection, we compare the estimators of location parameter via MRE. To do this, first, the MSE of estimators are computed, then, to find the superior estimator, we have considered the MRE of estimators. It is easy to find that,

$$
\operatorname{MSE}(\hat{\mu})=\frac{1}{\sigma^{2}} \quad \text { and } \quad M S E(\tilde{\mu})=\frac{1}{(n-1) \sigma^{2}} .
$$

Now, let us consider the MSE of $\tilde{\mu}^{P T}$, which could be rewritten as follows

$$
\begin{aligned}
M S E\left(\tilde{\mu}^{P T}\right)= & E\left[\left\{R_{1}-\frac{R_{n}-R_{1}}{n-1}-\left(R_{1}-\frac{R_{n}-R_{1}}{n-1}-\mu_{0}\right) I\left(A_{l}\right)-\mu\right\}^{2}\right] \\
= & \frac{1}{\sigma^{2}} E\left\{\left(U-\frac{V}{n-1}\right)^{2} I\left(\bar{A}_{l}\right)\right\}+\Delta_{l}^{2} E\left\{I\left(A_{l}\right)\right\} \\
= & \frac{1}{\sigma^{2}}\left[E\left\{U^{2} I\left(\bar{A}_{l}\right)+\frac{V^{2}}{(n-1)^{2}} I\left(\bar{A}_{l}\right)-\frac{2 U V}{n-1} I\left(\bar{A}_{l}\right)\right\}\right. \\
& \left.+\Delta_{l}^{2} E\left\{I\left(A_{l}\right)\right\}\right] .
\end{aligned}
$$

The last expression on the right hand side of (22) is given by

$$
\operatorname{Pr}\left\{A_{l}\right\}= \begin{cases}1-\frac{e^{\Delta_{l}}}{\lambda_{1}^{n-1}}, & \Delta_{l} \leqslant 0 \\ \rho_{n-1}\left(t_{1}, 1\right)-e^{\Delta_{l}} \rho_{n-1}\left(t_{1}, \lambda_{1}\right), & \Delta_{l}>0\end{cases}
$$

where $\rho_{n}(\cdot, \cdot)$ is defined in (7). Thus, calculating the $M S E\left(\tilde{\mu}^{P T}\right)$ reduce to finding the $E\left\{U^{2} I\left(\bar{A}_{l}\right)\right\}, E\left\{U V I\left(\bar{A}_{l}\right)\right\}$ and $E\left\{V^{2} I\left(\bar{A}_{l}\right)\right\}$. When $\Delta_{l}<0$, the expectation of $U^{2} I\left(\bar{A}_{l}\right)$ is given by

$$
\begin{aligned}
E\left\{U^{2} I\left(\bar{A}_{l}\right)\right\} & =\int_{0}^{\infty} \int_{\frac{f v}{n-1}-\Delta_{l}}^{\infty} u^{2} e^{-u} \frac{v^{n-2}}{\Gamma(n-1)} e^{-v} d u d v \\
& =\frac{e^{\Delta_{l}}\left(2-2 \Delta_{l}+\Delta_{l}^{2}\right)}{\lambda_{1}^{n-1}}+\frac{e^{\Delta_{l}} 2 f\left(1-\Delta_{l}\right)}{\lambda_{1}^{n}}+\frac{e^{\Delta_{l}}}{\lambda_{1}^{n+1}} \frac{n f^{2}}{(n-1)} .
\end{aligned}
$$


Moreover, for $\Delta_{l}>0$, the term $E\left\{U^{2} I\left(\bar{A}_{l}\right)\right\}$ is obtained as

$$
\begin{aligned}
E\left\{U^{2} I\left(\bar{A}_{l}\right)\right\}= & \int_{t_{1}}^{\infty} \int_{\frac{f v}{n-1}-\Delta_{l}}^{\infty} u^{2} e^{-u} \frac{v^{n-2}}{\Gamma(n-1)} e^{-v} d u d v \\
= & e^{\Delta_{l}}\left\{\left(2-2 \Delta_{l}+\Delta_{l}^{2}\right) \rho_{n-1}\left(t_{1}, \lambda_{1}\right)\right. \\
& \left.+2 f\left(1-\Delta_{l}\right) \rho_{n}\left(t_{1}, \lambda_{1}\right)+\frac{n f^{2} \rho_{n+1}\left(t_{1}, \lambda_{1}\right)}{(n-1)}\right\} .
\end{aligned}
$$

With similar method as was done for $U^{2} I\left(\bar{A}_{l}\right)$, we have

$$
E\left\{V^{2} I\left(\bar{A}_{l}\right)\right\}= \begin{cases}\frac{e^{\Delta_{l} n(n-1)}}{\lambda_{1}^{n+1}}, & \Delta_{l}<0 \\ e^{\Delta_{l}} n(n-1) \rho_{n+1}\left(t_{1}, \lambda_{1}\right), & \Delta_{l}>0\end{cases}
$$

and

$$
\begin{aligned}
& E\left\{U V I\left(\bar{A}_{l}\right)\right\}= \\
& \qquad \begin{cases}\frac{(n-1) e^{\Delta_{l}\left(1-\Delta_{l}\right)}}{\lambda_{1}^{n}}+\frac{n e^{\Delta_{l}}}{\lambda_{1}^{n+1}}, & \Delta_{l} \leqslant 0 ; \\
(n-1) e^{\Delta_{l}}\left(1-\Delta_{l}\right) \rho_{n}\left(t_{1}, \lambda_{1}\right)+n e^{\Delta_{l}} f \rho_{n+1}\left(t_{1}, \lambda_{1}\right), & \Delta_{l}>0 .\end{cases}
\end{aligned}
$$

Using (23), (24), (25), (26) and (27), we could find the MSE of $\tilde{\mu}^{P T}$. We have plotted the MRE of location estimators in Figures 7 and 8, which have similar behaviors to the scale parameter estimators.

\section{Illustrative Example}

In this section, we consider an example to illustrate the performance of the proposed PTE. Table 1 presents the times (in minutes) between 48 consecutive telephone calls to a company switchboard, presented by Castillo et al. (2005). They assumed that the times between 48 consecutive telephone calls followed exponential distribution, see Castillo et al. (2005) page 109. Table 2 contains the upper record extracted from the data in Table 1. The ML estimation of the scale parameter is $\hat{\sigma}=3.14$ and unbiased estimation is $\tilde{\sigma}=2.09$. Moreover, the estimations of location parameter are $\hat{\mu}=1.34$ and 

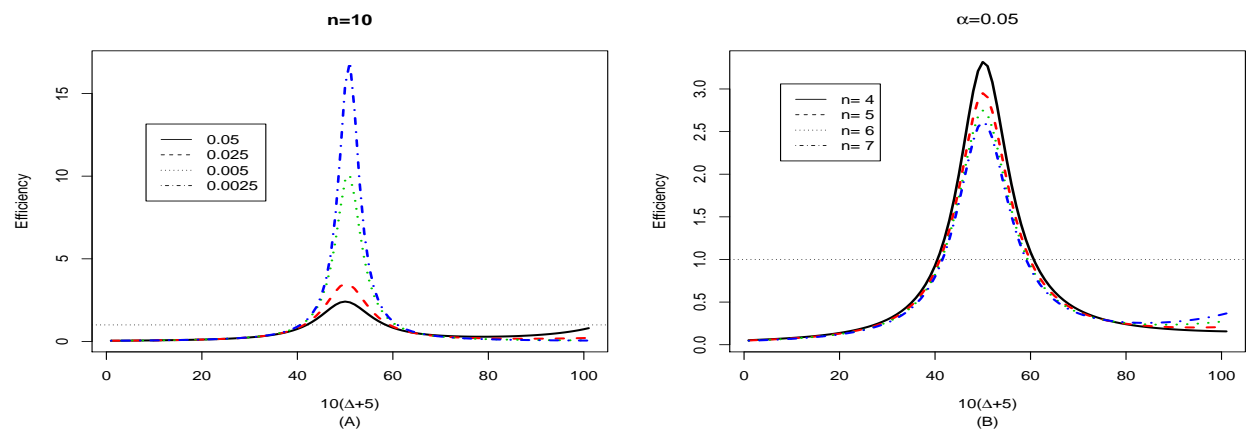

Figure 7. Efficiency of $\tilde{\mu}^{P T}$ relative to $\tilde{\mu}$ : (A) for fixed $n=10$, (B) for fixed $\alpha=0.05$.
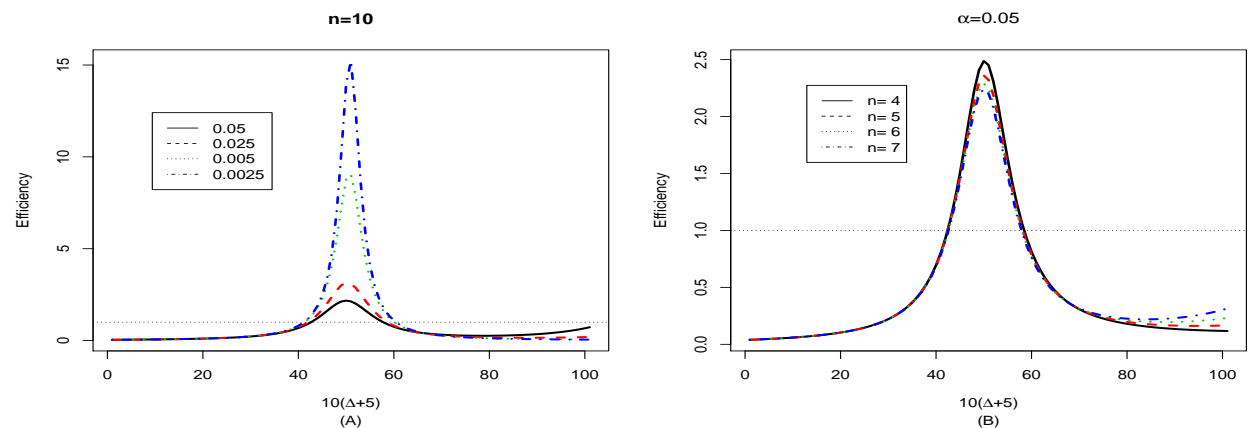

Figure 8. Efficiency of $\tilde{\mu}^{P T}$ relative to $\hat{\mu}$ : (A) for fixed $n=10$, (B) for fixed $\alpha=0.05$.

$\tilde{\mu}=0.99$. If there is an uncertain information about that is $\sigma_{0}=2.61$, then the PTE is chosen based on the result of following test

$$
H_{0}: \sigma=2.61 \text { vs } H_{1}: \sigma \neq 2.61
$$

Using Table 680 of Tate and Klett (1959) for $n=6$ and $\alpha=0.05$, the critical values are $a=4.7584$ and $b=24.3498$. Since

$$
4.7584<2 \sigma_{0}\left(R_{n}-R_{1}\right)=9.97<24.3498,
$$

there is no evidence to reject $H_{0}$, so the PTE is $\tilde{\sigma}^{P T}=2.61$. For the subjected PTE, we have computed MRE and GPC (PMC) in compare with UE (MLE). 
We consider two estimated values for $\Delta_{s}$ based on observed MLE $\left(\hat{\Delta}_{s}\right)$ and UE $\left(\tilde{\Delta}_{s}\right)$. Numerical results are presented in Table 3 . These results confirm the analytical claims in Sections 2 and 3.

Table 1. Times (in minutes) between 48 consecutive calls.

\begin{tabular}{llllllllllll}
\hline \hline 1.34 & 0.14 & 0.33 & 1.68 & 1.86 & 1.31 & 0.83 & 0.33 & 2.20 & 0.62 & 3.20 & 1.38 \\
0.96 & 0.28 & 0.44 & 0.59 & 0.25 & 0.51 & 1.61 & 1.85 & 0.47 & 0.41 & 1.46 & 0.09 \\
2.18 & 0.07 & 0.02 & 0.64 & 0.28 & 0.68 & 1.07 & 3.25 & 0.59 & 2.39 & 0.27 & 0.34 \\
2.18 & 0.41 & 1.08 & 0.57 & 0.35 & 0.69 & 0.25 & 0.57 & 1.90 & 0.56 & 0.09 & 0.28 \\
\hline
\end{tabular}

Table 2. Record values from times between 48 consecutive calls.

\begin{tabular}{ccccccc}
\hline \hline $\boldsymbol{i}$ & 1 & 2 & 3 & 4 & 5 & 6 \\
\hline $\boldsymbol{R}_{\boldsymbol{i}}$ & 1.34 & 1.68 & 1.86 & 2.20 & 3.20 & 3.25 \\
\hline
\end{tabular}

Table 3. MRE and PMC of PTE compare with MLE and UE for scale parameter.

\begin{tabular}{ccccc}
\hline & $M R E\left(\tilde{\sigma}^{P T}, \hat{\sigma}\right)$ & $M R E\left(\tilde{\sigma}^{P T}, \tilde{\sigma}\right)$ & $P M C\left(\tilde{\sigma}^{P T}, \hat{\sigma}\right)$ & $G P C\left(\tilde{\sigma}^{P T}, \tilde{\sigma}\right)$ \\
\hline$\hat{\Delta}_{s}=0.83$ & 3.70 & 1.23 & 0.7337 & 0.3002 \\
$\tilde{\Delta}_{s}=1.25$ & 3.83 & 1.27 & 0.5888 & 0.1279 \\
\hline
\end{tabular}

To find the PTE of location parameter, we have to conduct the following test

$$
H_{0}: \mu=0 \text { vs } H_{1}: \mu \neq 0 \text {. }
$$

In this case, the critical value is $f_{(2,10)}(0.95)=4.102$. Since

$$
\frac{(n-1) R_{1}}{R_{n}-R_{1}}=3.507<4.102,
$$

there is no evidence to reject $H_{0}$, which means that the PTE is zero. In Table 4, MRE and PMC of PTE are presented in compare with ML and UE.

Table 4. MRE and PMC of PTE compare with MLE and UE for location parameter.

\begin{tabular}{ccccc}
\hline \hline & $M R E\left(\tilde{\mu}^{P T}, \hat{\mu}\right)$ & $M R E\left(\tilde{\mu}^{P T}, \tilde{\mu}\right)$ & $P M C\left(\tilde{\mu}^{P T}, \hat{\mu}\right)$ & $G P C\left(\tilde{\mu}^{P T}, \tilde{\mu}\right)$ \\
\hline$\hat{\Delta}_{l}=4.2$ & 0.2 & 0.2411 & 0.541 & -0.269 \\
$\tilde{\Delta}_{l}=2.06$ & 0.286 & 0.343 & 0.325 & -0.670 \\
\hline
\end{tabular}


In comparison of $\tilde{\mu}^{P T}$ and $\tilde{\mu}$, both criteria, MRE and GPC, prefer $\tilde{\mu}$, so unbiased estimation is Pitman closer and more efficient than $\tilde{\mu}^{P T}$. However, in comparison of $\tilde{\mu}^{P T}$ and $\hat{\mu}$, when $\tilde{\Delta}_{l}=4.2, \mathrm{MRE}$ and PMC do not confirm each other. Now, this question arises for a practitioner that which estimator must be chosen in a practical situation. We respond that it depends on which criterion is more important for that user. If PMC is established as the most important criterion, then based on the results of Table 4 , the $\tilde{\mu}^{P T}$ must be considered for the purpose of estimation. On the other hand, if we consider the MRE criterion as the most important one, then we must prefer the $\hat{\mu}$. It may be noted that one numerical example does not tell us much more.

\section{Concluding Remarks}

In this article, the preliminary test estimator for the location and scale parameters of exponential distribution is introduced. Performance of the proposed estimator is investigated and compared with ML and UE by MSE and PMC criteria. It is noticeable that the results presented in this paper can be applied for other kind of ordered data such as censored data, $k$-record data. Moreover, let the underlying distribution be Parato with scale parameter $\mu$ and shape $\sigma$, then it is well-known that $\log (X)$ has exponential distribution with location $\log (\mu)$ and scale $\sigma$ which means that the MSE and PMC expressions of estimators of Pareto distribution are homogeneous to those in exponential distribution. Finally, since based on neither MSE nor PMC, PTE is uniformly superior to the corresponding competing estimators, ML and UE, further study may be needed to find the optimum significance level of the preliminary test, which leaves for further researches.

\section{Acknowledgement}

The authors would like to thank the referees for their constructive comments and suggestions which led to an improved version of this manuscript.

\section{References}

Ahmadi, J. and Balakrishnan, N. (2009). Pitman Closeness of Record Values to Population Quantiles. Statistics and Probability Letters, 79, 2037-2044.

Arnold, B.C., Balakrishnan, N. and Nagaraja, H.N. (1998). Records. John Wiley and Sons, New York. 
Arashi, M. and Tabatabaey, S.M.M. (2008). Stein-Type Improvement under Stochastic Constraints: Use of Multivariate Student-t Model in Regression. Statistics and Probability Letters, $\mathbf{7 8}, 2142-2153$.

Arashi, M. (2009). Preliminary Test Estimation of the Mean Vector under Balanced Loss Function. Journal of Statistical Research, 43, 55-65.

Arashi, M. (2012). Preliminary Test and Stein Estimators in Simultaneous Linear Equations. Linear Algebra and its Applications, 436, 1195-1211.

Baklizi, A. (2005). Preliminary Test Estimation in the Two Parameter Exponential Distribution with Time Censored Data. Applied Mathematics and Computation, 163, 639-643.

Balakrishnan, N., Davies, K.F., Keating, J.P. and Mason, L.R. (2011). Pitman Closeness, Monotonicity and Consistency of Best Linear Unbiased and Invariant Estimators for Exponential Distribution under Type II Censoring. Journal of Statistical Computation and Simulation, 81, 985-999.

Bancroft, T.A. (1944). On Biases in Estimation Due to the Use of the Preliminary Test of Significance. Annals of Mathematical Statistics, 15, 190-204.

Benda, N. (1996). Pre-Test Estimation and Design in the Linear Model. Journal of Statistical Planning and Inference, 52, 225-240.

Castillo, E., Hadi, A.S., Balakrishnan, N. and Sarabia, J.M. (2005). Extreme Value and Related Models with Applications in Engineering and Science, John Wiley and Sons, Hoboken.

Chiou, P. (1987). A Preliminary Test Estimator of Reliability in a Life-Testing Model. IEEE Transactions on Reliability, 36, 408-410.

Chiou, P. (1988). Shrinkage Estimation of Scale Parameter of the Extreme Value Distribution. IEEE Transactions on Reliability, 37, 370-374.

Han, C.P. and Bancroft, T.A. (1968). On Pooling Means when Variance is Unknown. Journal of the American Statistical Association, 63, 133-1342.

Hoque, Z., Khan, S. and Wesolowski, J. (2009). Performance of Preliminary Test Estimator under LINEX Loss Function. Communications in Statistics - Theory and Methods, 38, 252261.

Keating, J.P., Mason, R.L. and Sen, P.K. (1993). Pitman's Measure of Closeness: A Comparison of Statistical Estimators. Society for Industrial and Applied Mathematics, Philadelphia, Pennsylvania.

Khan, S. and Saleh, A.K.Md.E. (2001). On the Comparison of the pre-Test and Shrinkage Estimators for the Univariate Normal Mean. Statistical Papers, 42, 451-473. 
Kibria, B.M.G. and Saleh, A.K.Md.E. (2006). Optimum Critical Value for the pre-Test Estimators. Communications in Statistics- Theory and Methods, 35, 309-320.

Kibria, B.M.G. and Saleh, A.K.Md.E. (2010). Preliminary Test Estimation of the Parameters of Exponential and Pareto Distributions for Censored Samples. Statistical Papers, 51, 757773.

Kubokawa, T. (1989). Closer Estimators of a Common Mean in the Sense of Pitman. Annals of Institute of Statistical Mathematics, 41, 477-484.

Pitman, E.J.G. (1937). The "Closest" Estimate of Statistical Parameters. Proceedings of the Cambridge Philosophical Society, 33, 212-222.

Raqab, M. and Ahmadi, J. (2011). Pitman Closeness of Record Values from Two Sequences to Population Quantiles. Journal of Statistical Planning and Inference, 142, 855-862.

Saleh, A.K.Md.E. and Sen, P.K. (1991). Pitman-Closeness of Some Preliminary Test and Shrinkage Estimators. Communications in Statistics - Theory and Methods, 20, 3643-3657.

Saleh, A.K.Md.E. (2006). Theory of Preliminary Test and Stein-Type Estimations with Applications. Wiley, New York.

Tate, R.F. and Klett, G.W. (1959). Optimal Confidence Intervals for the Variance of a Normal Distribution. Journal of the American Statistical Association, 54, 674-682.

\section{Elham Mirfarah}

Department of Statistics, Ferdowsi University of Mashhad, Mashhad, Iran.

email: mirfarah.elham@gmail.com

\section{Jafar Ahmadi}

Department of Statistics, Ferdowsi University of Mashhad, Mashhad, Iran.

email: ahmadij@um.ac.ir 